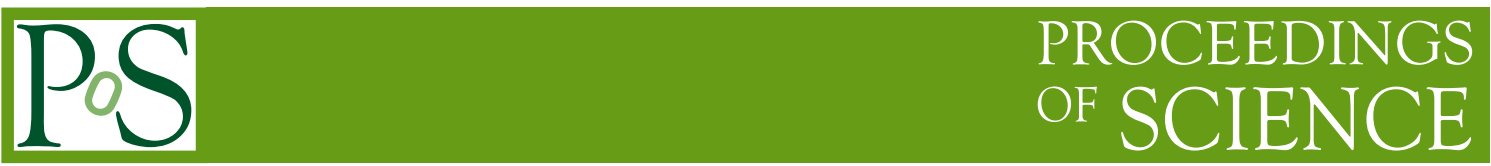

\title{
The EUSO@TurLab project
}

\author{
R. Caruso*, G. Contino, N. Guardone \\ Dipartimento di Fisica, Università di Catania \& INFN Catania, Italy \\ E-mail: rossella.caruso@ct.infn.it \\ M. Bertaina, G. Cotto, R. Forza, M. Manfrin, M. Mignone, H. Miyamoto, R. Mulas, \\ G. Suino, P. Tibaldi \\ Dipartimento di Fisica, Università di Torino \& INFN Torino, Italy
}

\section{for the JEM-EUSO Collaboration}

The EUSO@TurLab project is an on-going activity aiming to reproduce atmospheric and luminous conditions that JEM-EUSO will encounter on its orbits around the Earth, once it will be attached to the International Space Station (ISS). The TurLab laboratory, part of the Department of Physics of the University of Torino, is equipped with a rotating tank, normally used to perform fluidodynamics studies. In EUSO@Turlab project the facility is used to simulate different surface conditions with different optical characteristics, like snow, oceans, forests, glaciers, deserts, savannah in a very dark and rotating environment to test the response of JEM-EUSO's sensors and sensitivity. Moreover, it is possible to produce 'replica' of other types of luminous phenomena such as cosmic rays, meteors, city lights, lightnings etc. by using artificially controlled light sources. The detector is a simplified system consisting of a 25 pixel MultiAnode PhotoMultiplier Tube (MAPMT), a focusing lens and a read-out electronics using conventional modules. The experimental set-up currently in operation has been used to check the potential of the TurLab laboratory for the above purposes and the acquired data are used to test the concept of JEM-EUSO's trigger system. In this paper we describe the EUSO@TurLab project, present examples of the luminous conditions produced so far, as well as the results of the tests of the JEM-EUSO first level trigger applied to the data taken at TurLab.

The 34th International Cosmic Ray Conference,

30 July- 6 August, 2015

The Hague, The Netherlands

\footnotetext{
* Speaker.
} 


\section{Introduction}

In this work, we introduce the TurLab laboratory [1] (described in the next section) as a facility in preparatory steps of the JEM-EUSO project [2]. The aim of JEM-EUSO, that looks at the atmosphere from ISS downwards, is the detection of fluorescence and Cherenkov light produced by Extensive Air Showers (EAS) generated by Extreme Energy Cosmic Rays (EECRs, cosmic rays with energy $\mathrm{E}>5 \times 10^{19} \mathrm{eV}$ ) impinging on the Earth's atmosphere.

The atmosphere works as a detector for EECRs and, at the same time, is the main source of JEM-EUSO's noise, namely the night sky background. Possible atmospheric conditions creating fluctuations in the background luminosity are: a) variations of nightglow due to natural, slowly varying sources whose light is being reflected through Earth albedo (e.g. Moon in different phases, diffuse star light, airglow); b) artificial sources, e.g. city lights; c) Transient Luminous Events (TLE) in the lower and upper atmosphere (e.g. lightnings, ELVES and sprites); d) luminous phenomena, much slower than the speed of light, like meteors. Recognising the highly-variable luminous conditions of the atmosphere is the main task of the trigger system and simulations of different background conditions have been made to test and to implement and improve the trigger logic, written in $\mathrm{VHDL}^{1}$ code (feeding the acquired data in a computer in which it's running VHDL circuit simulator).

The use of TurLab gives the opportunity to reproduce experimentally variable luminous conditions and different possible light sources, recreating orbit sights and events on the JEM-EUSO scale. The TurLab represents an ideal condition for testing JEM-EUSO Focal Surface (FS) elements, electronics and trigger system at nearly real work conditions [4]. In the following we summarize the experimental set-up used for the above purposes. Details can be found in [5].

\section{The TurLab facility and the experimental apparatus}

The TurLab is located $15 \mathrm{~m}$ below ground level, in the Department of Physics, University of Torino. It is equipped with a $5 \mathrm{~m}$ diameter rotating tank (see Fig. 1) with a rotation period ranging from 12 seconds to 20 minutes; without artificial illumination the brightness in the room is much darker than the night sky background. In the EUSO@TurLab project we use the TurLab rotating tank with a series of different configurations to recreate JEM-EUSO's orbits and a MAPMT hung from the ceiling above the tank pointing towards nadir to mimic the JEM-EUSO telescope.

The possibility of changing the tank rotation speed allows events of different temporal duration to be reproduced with the same configuration and scenarios of different spatial extension to be represented as seen from the ISS. Most relevant possibility at TurLab is changing the light conditions and light sources. The latter ones are essentially of two kinds: 1) direct light emitting sources; 2) materials reflecting environmental light.

As light emitting sources we use a variety of instruments with the intent of reproducing different kinds of phenomena: a) LEDs inside tubes of different dimensions, to reproduce extended intense light directly pointing towards the MAPMT for simulating urban areas; b) an oscilloscope generating Lissajous curves for events such as meteors; c) LEDs driven by a pulse generator for

\footnotetext{
${ }^{1}$ VHDL (VHSIC Hardware Description Language, where VHSIC is the acronym of Very High Speed Integrated Circuit) is a hardware description language, born in 1987 by a project of the U.S Department of Defense. [3]
} 


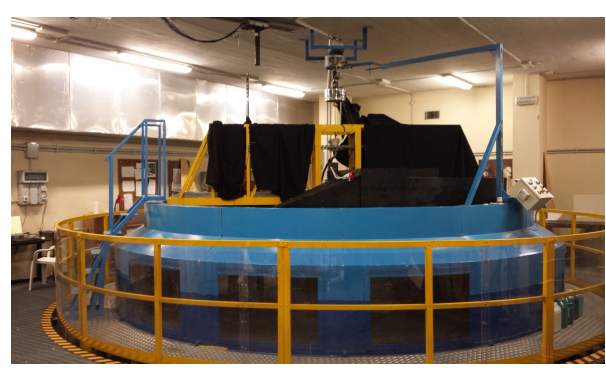

Figure 1: The TurLab rotating tank. The black tube on the ceiling is the collimator of the experimental set-up used to mimic the JEM-EUSO telescope.

fast luminous events such as lightnings; d) LEDs or optical fibers driven by Arduino [6] to get light pulses with $\mu$ s duration to reproduce light from cosmic ray EAS.

As materials reflecting environmental light, we use a variety of materials above the tank surface with the intent of reproducing Earth albedo: a) moss to mimic 'green' regions such as forests; b) glass dust for highly-reflectant surfaces such as glaciers or snow; c) sand for desert areas; d) bricks for land soils. We can increase the illumination in the tank room to get proportionally increasing irradiance using the same material. Presently, our environmental light sources are a computer screen beside the tank and a remote controlled LED over the tank.

EUSO@TurLab apparatus consists of: a) a (5×5) pixel MAPMT (Hamamatsu R8900-M25 [9]) looking towards nadir, free to move radially along two rails on the ceiling; b) a 1 " focusing lens, right in front of the MAPMT; c) 25 cables, $25 \mathrm{~m}$ long, connecting the MAPMT with electronics; d) standard NIM and CAMAC electronics (amplifiers, discriminators, scalers); e) a PC for acquiring measurements with LabVIEW [7] software.

The data are acquired on free-running mode: 1 frame every $\sim 30 \mathrm{~ms}$. The dead time of $30 \mathrm{~ms}$ is slightly variable during the run and it is mainly due to the data transfer time between electronics and computer. Offline, the data are processed through the first level trigger algorithm foreseen for the JEM-EUSO experiment. The electronic system, currently employed in the measurement, is very different from the JEM-EUSO one, however, it is based on the same detection principle of the single photon-counting [8]. Therefore, it can give a fair representation of the potentiality of EUSO@TurLab to mimic the experimental scenarios that JEM-EUSO is expected to encounter, and to tests, at a first level approximation, its trigger concept (the trigger algorithm is run offline).

The MAPMT with the lens has a Field of View $(\mathrm{FoV})$ of approximately $(0.14 \times 0.14) \mathrm{deg}^{2}$, i.e. each pixel sees $(5 \times 5) \mathrm{mm}^{2}$ at a $2 \mathrm{~m}$ distance. The solid angle is then of the order of $10^{-6} \mathrm{sr}$, the same as the solid angle of a pixel in JEM-EUSO. It turns out that the ratio of the pixel's FoV at TurLab and in JEM-EUSO is $(5 \mathrm{~mm} / 500 \mathrm{~m}) \sim 10^{-5}$, which is the same as the ratio of the tank speed compared to the ISS one, if the tank makes a full rotation in $\sim 3$ minutes and the telescope is located at $2 \mathrm{~m}$ from the centre of the tank.

The electronics has a double-pulse resolution of $400 \mathrm{~ns}$; the Gate Time Unit (GTU) is $40 \mu \mathrm{s}$ to have a ratio of the order of JEM-EUSO's one: $10 \mathrm{~ns}$ double-pulse resolution in a $2.5 \mu$ s GTU.

The MAPMT has a 4:1 pixel-by-pixel non uniformity in Detection Efficiency (DE) ${ }^{2}$. The M64

\footnotetext{
${ }^{2}$ The detection efficiency is the ratio of the detected signal to the input signal of a PMT. In photon counting this is expressed as the product of the photocatode quantum efficiency and the collection efficiency. [10]
} 
MAPMT employed in JEM-EUSO is expected to have a better performance [11].

\section{Performed Tests}

The advantage of using the above system instead of dedicated simulations is that it introduces naturally all sorts of noise and fluctuations which are difficult to model or to be foreseen in computer simulations.

In the Fig. 2 there are the MAPMT counts during a complete 10 minute rotation of the tank. Different configurations are reproduced. The environmental light by the LED over the tank is the same for the two curves. The blue curve refers to the signal acquired by the MAPMT when it sees the rotating tank equipped with a collection of different materials and direct light sources laying on the surface of the tank, while the green one refers to the signal acquired by the MAPMT when it sees the rotating tank without any light sources.

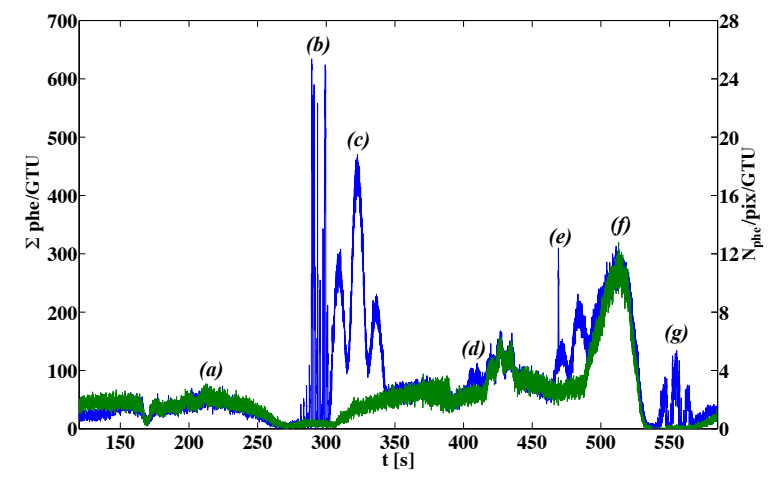

Figure 2: A 10 minutes rotation of the tank equipped without (green) and with (blue) different sources: a) moss; b) 10 LED strip controlled by Arduino; c) mirror; d) bricks; e) glass dust; f) sand; g) oscilloscope. See text for details.

Looking at results in Fig. 2, one can see how the tank itself looks like a source of light when it is sufficiently illuminated by environmental light, due to structures that are part of the tank: there are metal bars, a footbridge and a wooden structure created for other experiments. All of these structures can be covered/painted with black to reduce their effect on luminosity. Some of them can be removed if necessary to reach a more uniform response. On the other hand they constitute natural inhomogeneities useful to test the capability of the system to adjust the thresholds for trigger purposes.

The limited number of pixels used in the performed tests (25) is only $~ 10 \%$ of 1 Elementary Cell of JEM-EUSO (EC) which is formed by 4 MAPMTs of $(8 \times 8)$ pixels, for a total of 256 pixels. The different numbers of pixels used with respect of the JEM-EUSO EC represents a minor problem when we reproduce a track of an almost vertical cosmic ray, because the signal is detected only by a few pixels, whereas, in case of cities or simply the background data, it must be taken into account and all pixels need to be reproduced because the first level trigger is applied to EC data.

In the last case we resample randomly in time the data of the real pixels and for each real pixel we attribute such values to 9 virtual pixels. Therefore the data in the 10 pixels $(9$ virtual pixels +1 real pixel) have the same DE. At a first approximation level this is acceptable, keeping in mind that much longer databases are needed to make sure that the fluctuations are properly reproduced. 


\subsection{Background and EAS-like events}

A detailed description of the first level trigger is reported in [4]. Here we summarize the main features. The first level trigger operates at EC level. It collects the signal in boxes of $(3 \times 3)$ near-by pixels for a few consecutive GTUs. If this signal is higher than a preset threshold, a trigger flag is issued. The thresholds to set the trigger flag of the excess of signal at pixel level and in the boxes depend on the average background level. Such background is calculated by averaging the number of counts in $(2 \times 4)$ wide subsamples of the EC for 128 GTUs. In this way every $320 \mu \mathrm{s}$ the trigger parameters are defined and applied for the next $320 \mu \mathrm{s}$.

Long runs of constant or varying background have been performed to collect enough background data. LED crossing the M25 MAPMT or Arduino set-up have been used to reproduce cosmic rays.
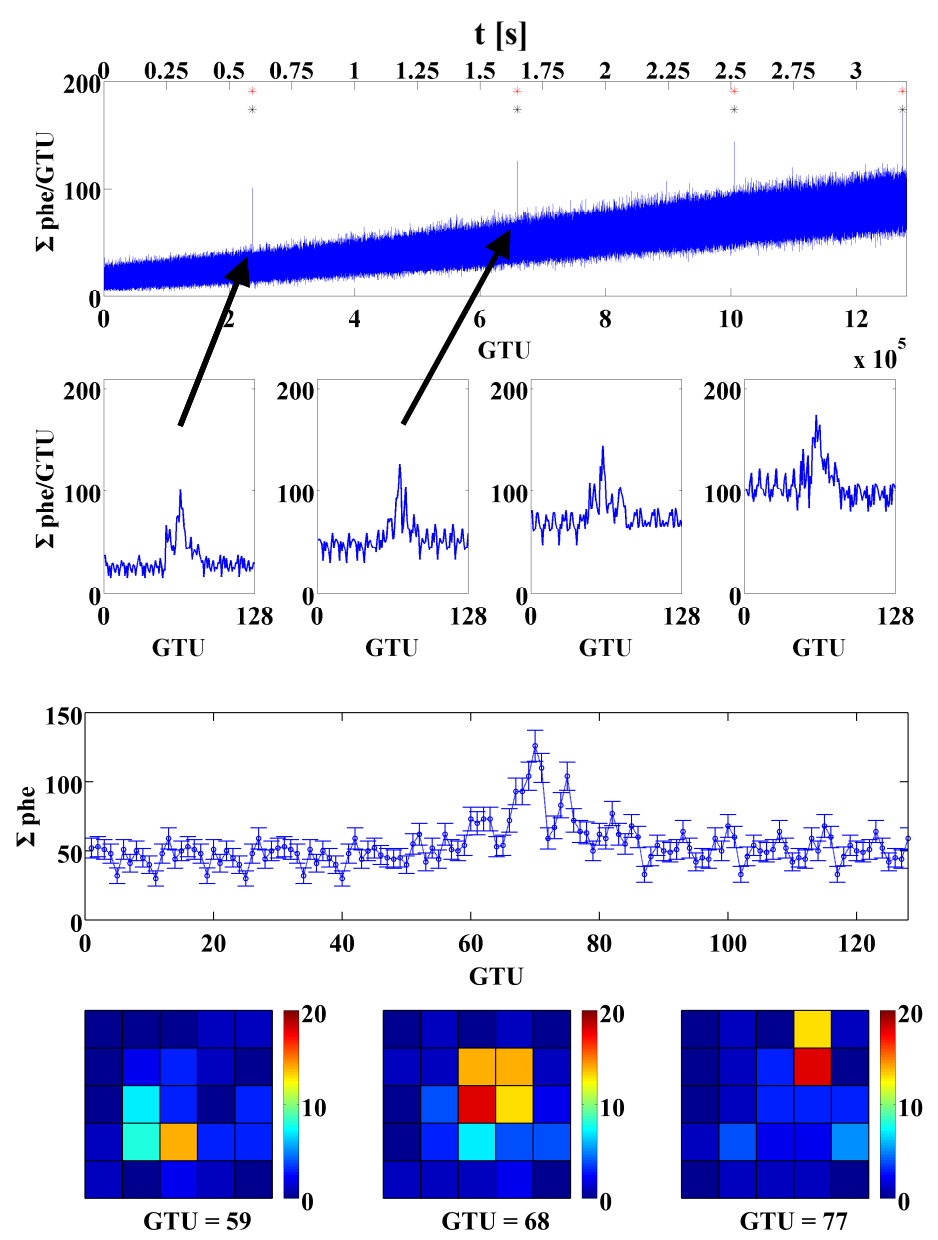

Figure 3: Top half: reproduction of 4 EECR-like events in 3 seconds of variable background data (see arrows for the first two ones). Stars indicate the time at which the trigger in the VHDL code was fired. Higher panel represents the whole set of data. In the lower panel there is the zoom of the four events.

Bottom half: zoom of the second event. Higher panel is a more detailed view of the second event, in which there is the light profile in 128 GTUs with the light excess due to the fired LEDs around GTU 70. In the lower panel there are the snapshots of 3 different GTUs during the event occurrence. 
In the Fig. 3 there is an example of such type of events. Three seconds of data with variable background between $\sim 1-4$ counts/pixel/GTU and 4 cosmic ray events are reported. Each EAS track lasts 35-40 GTUs. A zoom on the 128 GTUs around the events is shown on top of the figure. The stars indicate the time at which a trigger occurred. It is always in time with the EAS event. The threshold adjustment on the variable background avoids triggers on background. The black stars assume that the JEM-EUSO electronics system doesn't have dead time. The red stars on the other hand assume that, after a trigger, the data trasfer to next trigger level requires $20 \mathrm{~ms}$; in such fraction of time, therefore, the first level trigger is basically not operating. The EAS events reproduced artificially have comparable shape and intensity of a $2 \times 10^{20} \mathrm{eV}$ event detected by JEM-EUSO. The background level applied to the events corresponds for JEM-EUSO to $\Phi=500$ - 2000 photons $\mathrm{m}^{-2} \mathrm{~ns}^{-1} \mathrm{sr}^{-1}$ for clear sky, which is the assumed condition for determining the exposure curve reported in [12] and successive publications.

\subsection{Lightning-like events}

In the Fig. 4, the left part is the case of a lightning-like signal as it is expected to be seen by the JEM-EUSO EC when the lightning is in its FoV. In the TurLab, the events were generated using the same technique previously described. The light intensity has been artificially changed to mimic the response of JEM-EUSO's protection system. A real lightning is assumed to last $\sim 40 \mathrm{~ms}$ and starts $\sim 13 \mathrm{~ms}$ after the beginning of the acquisition time. Light increases for $\sim 12$ $\mathrm{ms}$, reaches a maximum and then decreases, disappearing $\sim 25 \mathrm{~ms}$ after the maximum. After a while from the beginning of the event, the intensity of the detected signal is so high that JEMEUSO protection system is activated [13]. At this time, with a couple of GTUs delay, the DE of the PMT is reduced by a factor of 100 by the protection system and the intensity of the signal suddenly decreases; however, the light emission is still increasing, therefore, the detected signal starts to increase again but slower in time because of the much lower DE. Around $12 \mathrm{~ms}$ from the beginning, the lightning has reached its maximum and light tends to decrease, then after $25 \mathrm{~ms}$ the lightning is over; nevertheless DE is still at reduced level and the signal on the EC is extremely weak. At about $100 \mathrm{~ms}$ from the beginning, the standard photon DE is restored and a sudden increase of the signal occurs. The automatic adjustment of the thresholds on varying background follows quite nicely the event.

In the TurLab we have reproduced artificially a lightning with the same temporal characteristics described before. By analyzing the data, we identify a trigger just at the beginning of the event, followed by a second one $\sim 12 \mathrm{~ms}$ afterwards. By assuming $\sim 20 \mathrm{~ms}$ dead time among events, this second trigger would be missed (only the black star is shown). A random trigger occurs during the darkest part of the event, still compatible with the rate of random triggers. The last trigger occurs again when the baseline goes back to the normal value due to the sudden transition of the DE. In general, the trigger logic reacts to the light sequence, as expected. As lightnings occur sparsely, this is not a big issue for the system. In reality, lightning measurements are one of the exploratory objectives of JEM-EUSO, therefore the activation of the protection system is a confirmation of the presence of an extremely bright light source that could be used to start a special acquisition for lightning events. 

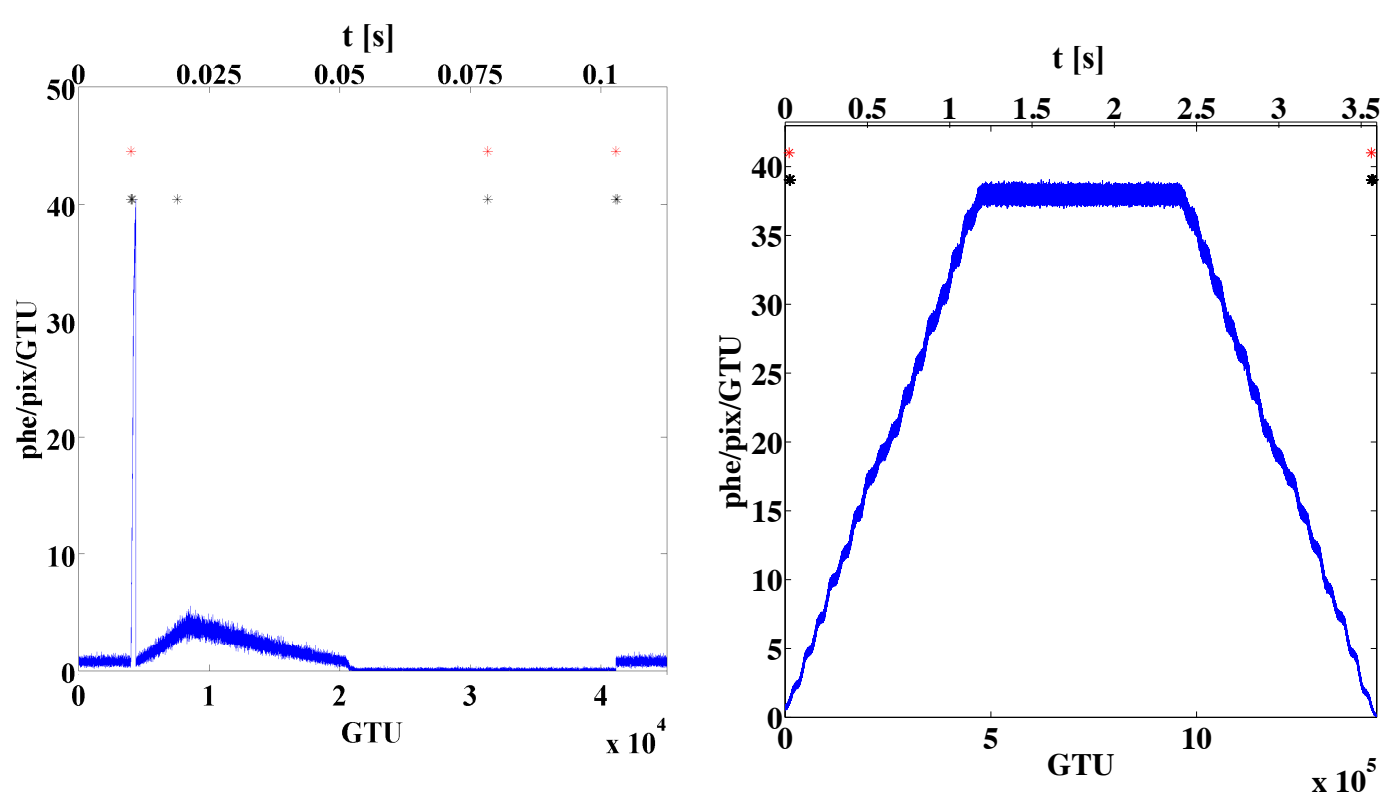

Figure 4: Left: reproduction of a lightning as seen by an EC in the FoV of the lightning. Stars indicate the time at which the trigger in the VHDL code was fired. See the text for a detailed explanation of the light pattern. Right: reproduction of a city-like light profile crossing an EC in the FoV.

\subsection{City-like events}

Finally, city-like events are described in the following. They are the slowest type of events as the light evolution occurs at ISS speed. Right part of Fig. 4 is the light profile of an urban area with a linear extension of $\sim 15 \mathrm{~km}$, which crosses perpendicularly one edge of the EC-FoV. The signal increases up to a factor $\sim 40$ compared to the standard nightglow conditions. Such an increase is compatible with previous experimental measurements [14].

Each pixel is fully crossed by the light source in $\sim 70 \mathrm{~ms}$ due to the ISS speed. In this case the trigger algorithm works pretty well since only two spurious triggers occur, due to the sharp transit of light emitting source (see section 2) in the FoV of the PMT. In general, the automatic evaluation of the average background coupled with the system that automatically adjusts the thresholds to keep the rate of triggers on background fluctuations below $1 \mathrm{~Hz} / \mathrm{EC}$ even in case of slow background variations, allows controlling the rate of fake triggers. Moreover, when the light background level increases above the trigger threshold of 6.3 counts/pixel/GTU, the trigger doesn't fire anymore.

\section{Conclusions}

The measurements presented in this paper represent the first successful steps of an on-going project aiming at using TurLab as a facility to mimic experimentally transient lights that JEMEUSO is expected to see while measuring from the ISS. We plan to improve further our set-up to reach a more realistic representation of the JEM-EUSO system and of the physical processes it will monitor.

The data taken so far have been used to validate the logic of the first level of its trigger system. The system that automatically adjusts the thresholds is effective. The first level trigger detects 
EAS-like events with light intensities comparable to those JEM-EUSO would observe in the expected energy range and in presence of the expected night sky background intensities. These results strengthen those obtained in [12] and successive publications, as they show that the trigger concept adopted in those simulations can be effectively implemented in hardware.

The first level trigger has shown to be quite effective in rejecting city-like and lightning type of events. Only few spurious triggers occur. The examples shown in this paper constitute a subsample of the tests, that are on going at the TurLab facility, to further check the robustness of the algorithm to a larger variety of phenomena. Moreover, in parallel the trigger algorithm is tested using EUSO-Balloon [4] data.

Currently the photo-detection and the electronic systems are being changed to the JEM-EUSO's one to test a fully equipped EC of JEM-EUSO's focal surface. TurLab could be used in future to test prototypes and precursors of JEM-EUSO, like mini-EUSO [15].

Acknowledgment: This work was partially supported by Basic Science Interdisciplinary Research Projects of RIKEN and JSPS KAKENHI Grant (22340063, 23340081, and 24244042), by the Italian Ministry of Foreign Affairs and International Cooperation, by the 'Helmholtz Alliance for Astroparticle Physics HAP' funded by the Initiative and Networking Fund of the Helmholtz Association, Germany, and by Slovak Academy of Sciences MVTS JEM-EUSO as well as VEGA grant agency project 2/0076/13. Russia is supported by the Russian Foundation for Basic Research Grant No 13-02-12175-ofi-m. The Spanish Consortium involved in the JEM-EUSO Space Mission is funded by MICINN \& MINECO under the Space Program projects: AYA2009-06037-E/AYA, AYA-ESP2010-19082, AYA-ESP2011-29489C03, AYA-ESP2012-39115-C03, AYA-ESP2013-47816-C4, MINECO/FEDER-UNAH13-4E-2741, CSD2009-00064 (Consolider MULTIDARK) and by Comunidad de Madrid (CAM) under projects S2009/ESP-1496 \& S2013/ICE-2822.

\section{References}

[1] http://www.turlab.ph.unito.it

[2] T. Ebisuzaki et al, Adv. Sp. Res. 53 (2014) 1499.

[3] http://www.vhdl.org/

[4] G. Suino et al. (JEM-EUSO Coll.), Proc. 34th Int. Cosmic Ray Conf. (Den Haag), \#0925 (2015).

[5] M. Bertaina et al. (JEM-EUSO Coll.), EPJ Web of Conferences 89 (2015) 03003.

[6] http://www.arduino.cc

[7] http://www.ni.com/labview

[8] D.V. O'Connor, D. Phillips, Time correlated Single Photon Counting, Academic Press Inc. ISBN 0-12-524140-2 (2012)

[9] Y. Kawasaki et al., Nucl. Instr. \& Meth. A 564 (2006) 378.

[10] Hamamatsu, Photomultiplier Tubes: Basics and Applications (Third Edition)

[11] Y. Kawasaki et al., Proc. 32th Int. Cosmic Ray Conf. (Beijing), 3 (2011) 96; arXiv:1204.5065.

[12] J.H. Adams Jr. et al. (JEM-EUSO Coll.), Astrop. Phys. 44 (2013) 76.

[13] S. Bacholle et al., Proc. 34th Int. Cosmic Ray Conf. (Den Haag), \#1364 (2015).

[14] O. Catalano et al, Nucl. Instr. \& Meth. A 480 (2002) 547.

[15] http://jem-euso.roma2.infn.it/?page_id=818 